\title{
SEIS ISLAS TEMPORALES EN STILL - THE ECONOMY OF WAITING
}

\section{Miguel Ángel Melgares Calzado}

\author{
Amsterdam University of the Arts \\ Universidad de Granada. Doctorando
}

\section{Resumen}

El artículo se centra en el análisis de la instalación performática Still - The Economy of Waiting, obra del artista alemán Julian Hetzel. En dicho proyecto se abordan ciertas problemáticas asociadas a los mecanismos en la producción laboral y las éticas del trabajo. Tomando como punto de partida modelos de producción posfordistas, Hetzel observa la actual transformación de la idea de "trabajo" en la idea de "ocupación". Mientras que la idea de "labor y trabajo" han estado orientadas hacia un modelo productivo que persigue resultados y objetivos específicos, la idea de "ocupación" se centra en la actividad como proceso, es decir, su finalidad no es otra que la de permanecer ocupado, planteando con ello un contra-modelo temporal con el que generar una brecha en el flujo de la circulación capitalista.

Palabras clave: PERFORMANCE; TIEMPO; LABOR; OCUPACIÓN; POSFORDISMO

\section{SIX TEMPORAL ISLAND AT STILL - THE ECONOMY OF WAITING}

\section{Abstract}

This article focuses on the analysis of the performative installation Still - The Economy of Waiting, work by German artist Julian Hetzel. This project addresses specific issues related to the mechanisms in labour productivity and work ethics. Taking as a starting point post-Fordist production models, Hetzel observes the current transformation of the idea of "work" into the notion of "occupation". While "labour and work" concepts have been oriented towards a productive model that pursues specific results and objectives, the concept "occupation" focuses on activity as a process, that is, its purpose is none other than to remain busy, thereby raising a temporary counter-model with which to generate a gap in the flow of capitalist circulation.

Keywords: PERFORMANCE; TIME; LABOR; OCCUPATION; POSTFORDISM

\footnotetext{
Melgares Calzado, Miguel Ángel. 2019. "Seis islas temporales en Still The Economy of Waiting“. AusArt 7 (2): 137-152. D0I: 10.1387/ausart.21127
}

\section{AUSART}




\section{INTRODUCCIÓN}

Nuestra conciencia, percepción y construcción del tiempo ha evolucionado en paralelo al devenir de los cambios históricos, sociales, políticos, y especialmente económicos. De entre las múltiples temporalidades que se producen en la performance, las prácticas escénicas han estado supeditadas a la idea de la construcción de una comunidad temporal, reunida para disfrutar colectivamente de una obra. Por el contrario, otras formas artísticas, desde la literatura a las artes visuales, han seguido un principio de emancipación individual, marcando su devenir cultural por la capacidad de decidir individualmente el tiempo de consumo temporal de una obra. En el contexto de la relación entre el texto dramático y el teatro, Lehmann ([1999] 2017, 304) nos expone el dilema del siguiente modo: "Mientras que el texto deja al lector la elección de llevar a cabo su lectura más despacio, más deprisa, de repetir y hacer pausas, el tiempo específico de la realización escénica, con su ritmo particular y su propia dramaturgia (velocidad del discurso y de la actuación, duración, pausas de hablar, etc.) viene determinado por la obra".

En cierto sentido, a partir de esta formulación podríamos entender que en esta dicotomía se evidencian las diferencias de dos tipos de usos temporales, que podríamos definir como un tiempo objetivo y un tiempo subjetivo, o quizás podríamos definirlos como un tiempo que se construye en el pensamiento, y otro bien diferente que cuelga de la pared. A principios del siglo XX el filósofo francés Henri Bergson introduce en Evolución creativa (1907) un tiempo que trata de plantear una alternativa a la linealidad, segmentación y organización temporal (propias de un capitalismo industrial), defendiendo el concepto de "duración", con el objetivo de moldear a través de su fluidez y movilidad la lógica lineal del tiempo. Como expone Miguel Ángel Hernández-Navarro (2008, 9): "Si algo quedó claro a lo largo de la segunda mitad del siglo pasado es que la historia no puede ser pensada -al menos sólo pensaba-diacrónicamente. [...] Existen saltos, discontinuidades, azares, confrontaciones y contradicciones. [...] Pasado, presente y futuro - experiencia, acción y expectativa- no sólo seducen diacrónicamente, sino también de un modo sincrónico. No sólo uno detrás de otro, sino todos al mismo tiempo, anudándose en una simultaneidad temporal".

Con el objetivo de ejemplificar ciertas estrategias artísticas en el uso del tiempo en el performance contemporáneo, en las siguientes páginas analizaremos una propuesta que nos ofrece modelos temporales singulares, construidos a partir del concepto de duración como estructura temporal performática. El 
trabajo del que nos estamos haciendo eco es Still - The Economy of Waiting (2014) del artista alemán Julian Hetzel. Dicha propuesta plantea una flexibilización temporal para con la práctica posdisciplinaria del performance, donde consigue romper con cualquier binarismo discursivo en torno a lo temporal. El uso del tiempo en este performance no solo funciona como un vehículo a través de cuál desarrollar la propuesta, sino que el concepto de "tiempo" busca tanto la producción de experiencias como el desarrollar el pensamiento crítico en el público.

\section{STILL. THE ECONOMY OF WAITING}

Presentado por primera vez en el contexto de SPRING 2014 en la ciudad holandesa de Utrecht, Hetzel realizó Still - The Economy of Waiting, una instalación performativa con la que trataba de analizar la relación entre los conceptos de 'esperar' y 'trabajar'. Elaborada a partir de una serie de contenedores de transporte comercial situados en el centro de una plaza pública —Janskerkhof-, la instalación despliega un viaje poliédrico donde la relación entre ficción y realidad queda suspendida. A lo largo del recorrido, el espectador se encuentra con seis islas temporales. Seis habitáculos, seis situaciones, seis encuentros, seis temporalidades.

El punto de partida de Still - The Economy of Waiting radica en el interés de Hetzel por la transformación en los modelos de producción laboral. El concepto de trabajo generalmente se defiende como una "obligación ética colectiva" (Weeks 2013, 149). De hecho, el trabajo es uno de los ejes fundamentales a través del cual los individuos se integran no solo en un sistema económico, sino también dentro de modos de cooperación familiar, social y políticos. Es más, el trabajo aparece en el centro de la construcción de la identidad personal. Sin embargo, y mientras que el devenir de los tiempos parece transformar esta concepción del trabajo, Hetzel plantea cómo las éticas laborales deberían ser igualmente cuestionadas. Analizando los modelos de producción posfordistas, Hetzel observa la actual transformación de la idea de "trabajo" en la idea de "ocupación". Mientras que la idea de 'labor y trabajo' están orientadas hacia un modelo productivo que persigue resultados y objetivos específicos, la idea de 'ocupación' se centra en la actividad como proceso, es decir, su finalidad es el permanecer ocupado. Tal y como planteara Hito Steyerl (2016), entendemos que la ocupación no dispone de un marco temporal específico, excepto 
el mero hecho de pasar el tiempo: “El cambio del 'trabajo' por 'ocupación' se aplica en las áreas más diferentes de la actividad cotidiana contemporánea. Marca una transición mucho mayor que el cambio a menudo descrito de una economía fordista a posfordista. En lugar de entenderse como un mecanismo ganancial, es visto como una forma de consumir tiempo y recursos. Acentúa claramente el paso de una economía basada en la producción a una economía alimentada por el desperdicio, desde el tiempo progresivo hasta el tiempo gastado o incluso inactivo, desde un espacio definido por divisiones claras hasta un territorio enredado y complejo. Quizás lo más importante: la ocupación no es un medio para alcanzar un fin, como lo es el trabajo tradicional. La ocupación es en muchos casos un fin en sí mismo"'.

Es precisamente en este contexto de sociedad posfordista, donde Julian Hetzel propone con la obra Still - The Economy of Waiting un contra-modelo temporal con el que generar una brecha en el flujo de la circulación capitalista. Para ello, Hetzel nos invita a recorrer un universo creativo singular, que pese a generar un espacio y temporalidad paralelas, permanece íntimamente conectado a la realidad política que sucede más allá de las finas paredes de los contenedores industriales donde se desarrolla el proyecto.

Cuando llegamos a Janskerkhof, una de las plazas históricas de la ciudad, el sonido es lo primero que notamos. A través de un sistema de megafonía, una persona - con un acento marcadamente asiático - nos informa de manera puntual de la hora exacta. Cada diez segundos la persona repite un mantra en constante transformación. 6 veces por minuto, 360 veces por hora y 2880 en las ocho horas que la instalación permanece diariamente abierta al público, una voz nos informa:

At the next tone the time will be sixteen hours, twenty minutes and thirty seconds. "Ding"

At the next tone the time will be sixteen hours, twenty minutes and forty seconds. "Ding"

At the next tone the time will be sixteen hours, twenty minutes and fifty seconds. "Ding"

At the next tone the time will be sixteen hours and twenty-one minutes. "Ding"

At the next tone the time will be sixteen hours, twenty-one minutes and ten seconds. "Ding"

At the next tone the time will be sixteen hours, twenty-one minutes and twenty seconds. "Ding" 


\section{ESPACIOS - CONTENEDORES - TIEMPOS}

En la plaza se disponen un conjunto de contenedores - ocho para ser exactos- que conforman una especie de laberinto arquitectónico efímero. Estos grandes contenedores de mercancías varados en el espacio público, ponen en perspectiva el título del proyecto². Diseñados para resistir largos viajes marítimos, los contenedores se apilan en los puertos del mundo esperando para ser transportados a sus destinos. Desde su funcionalidad, estandarización y neutralidad, estos contenedores constituyen un elemento central en el paisaje mercantil globalizado.

\section{Room 1. YOUTUBe CINEMA}

Hetzel nos propone un viaje inmersivo donde el espectador gestiona la temporalidad de su visita en primera persona, negociando la relación entre el tiempo productivo y el tiempo contemplativo que Hetzel nos propone como experiencia performática. La entrada a la instalación se produce individualmente, respetando un intervalo de unos cinco minutos entre los visitantes. Dicha entrada se produce por uno de los contenedores, accediendo directamente a la primera de las salas. El interior ha sido modificado para habilitar espacios que podrían asimilarse a los de una galería de arte. El rótulo con el título de la instalación, el texto explicativo integrado en la pared y la iluminación nos invitan a investigar en el contenido de la experiencia. En ese mismo espacio nos encontramos con una primera instalación que funciona como un pequeño gesto artístico con el que nos sentimos fácilmente identificados. Una pantalla reproduce la imagen de la página web de Youtube en el momento en que se está cargando un video. Este momento se reproduce en un bucle que no encuentra salida. El público espera educadamente sentados en el banco a que el video se cargue, hasta que entienden que la imagen del video cargándose es el video en sí mismo.

\section{Room 2. Gallery}

Tras este guiño, nos adentramos en el siguiente de los contenedores, donde nos encontramos con un guardia de seguridad custodiando una obra de arte, para ser más exactos un retrato de gran formato. Como ocurriera en el anterior espacio, este contenedor ha sido cuidadosamente transformado, utilizando todos los códigos estéticos y espaciales que nos hagan sentir en una galería de arte contemporáneo. Hetzel se interesa por la presencia física de los guar- 
dias de seguridad en los espacios expositivos, cuyo trabajo esencialmente consiste en estar presentes a la espera de visitantes. En el proceso de selección de los guardias de seguridad, Hetzel busca personas de edad avanzada que nos ayuden a empatizar con la idea de un trabajo tedioso, invisible y repetitivo. Sin embargo, Hetzel nos propone un interesante giro. El retrato que el guardia está custodiando es una obra hiperrealista al óleo cuyo protagonista es el propio guardia de seguridad, vestido con el mismo uniforme, sentado en la misma silla, y retratado en el mismo espacio donde se está desarrollando la acción. La imagen del retrato no posee la solemnidad de un retrato formal, sino que trata de capturar la humanidad imperfecta del retratado. Junto al retrato aparece una descripción de la obra:

\section{Seated Guard}

Security Guard, Painting (Oil on canvas, 180 x $120 \mathrm{~cm}$ ), 2014. Oil painting made by an anonymous painter working for dafenvillageonline.com, Dafen, Shenzhen, China.

The painting is a replica of a digital photo snapshot of the security guard Joop Kortland, employed for STILL in agreement with Securitas $\mathrm{GmbH}$. The original photo was taken by Julian Hetzel on April 10th 2014 in Utrecht, The Netherlands, using a Nikon G1 camera. After being color-corrected in Adobe Photoshop, it has been sent via e-mail by STILL's production manager Lyndsey Housden, as a jpeg-file $(6,3 \mathrm{MB}, 2848 \times 4288 \mathrm{px}, 300 \mathrm{dpi})$ to the customer request coordinator of dafenvillageonline.com on April 17th, 2014. The picture has been hand painted, within 15 working days, by an anonymous painter working for the company. After consulting for approval, the final product was delivered by airmail to Utrecht within five working days.

En una clara conexión con el trabajo conceptual de Joseph Kosuth One and Three Chairs (1965), Hetzel nos propone un interesante giro en la estrategia tautológica y formal de Kosuth ${ }^{3}$. Mientras que el triple código objetual, visual y verbal propuesto por Kosuth nos aproxima a los mecanismos de presentación-representación-conceptualización del arte, con su instalación Hetzel primero consigue humanizar el arte conceptual, para inmediatamente politizar los procesos productivos del arte. La deslocalización y anonimato de la persona que ejecuta el retrato, nos hace pensar sobre el concepto de autoría, al mismo tiempo que nos hace reflexionar en el proceso de creación mecánico e impersonal tras la producción de dicho retrato. 
Sin embargo, el engranaje de relaciones laborales que esta propuesta encierra, mas allá de exponer una alienación de los mecanismos productivos de una

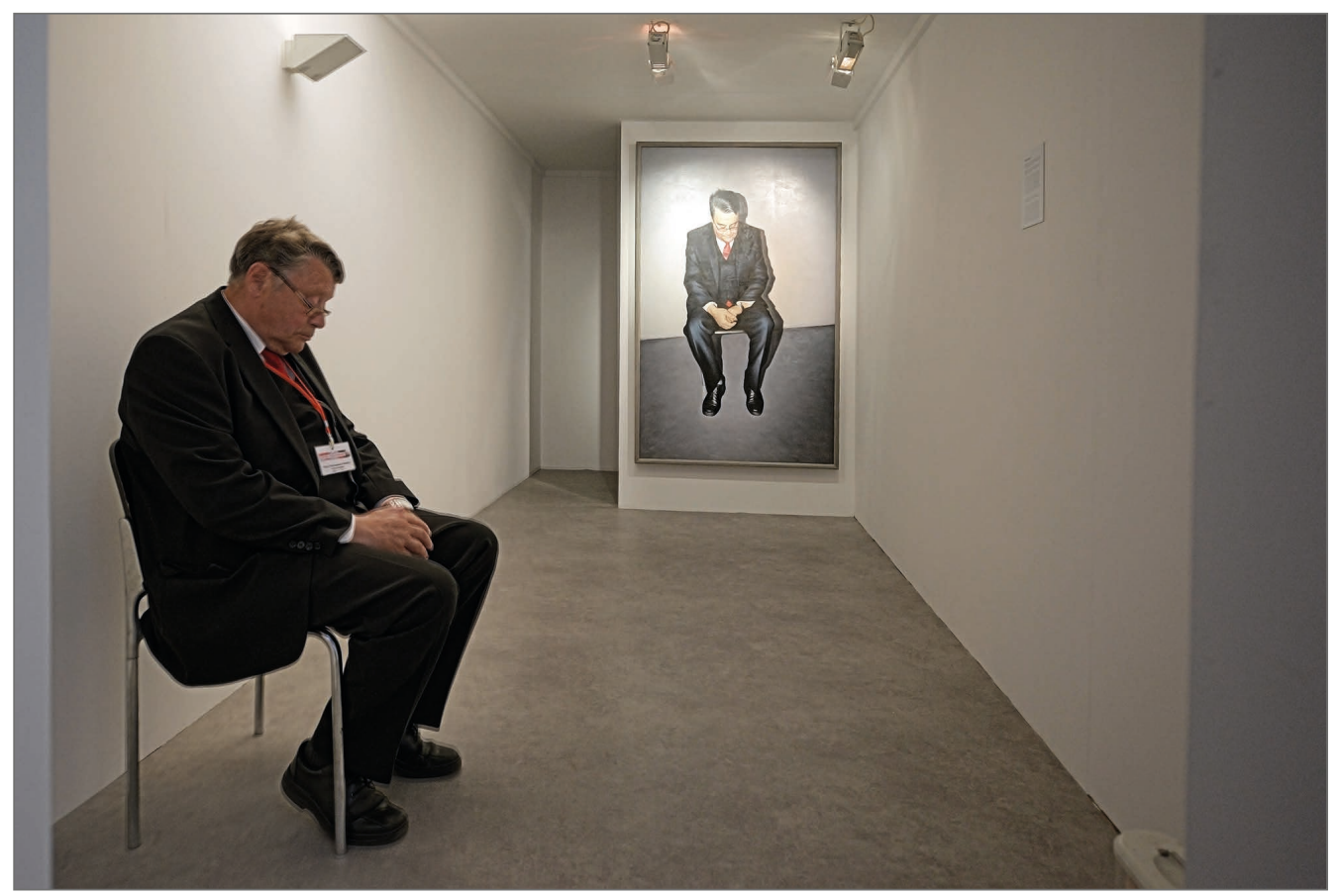

Fig. 1. Still —The Economy of Waiting, (2014). Julian Hetzel. Imagen de la instalación. ( Andreas Etter.

economía globalizada, también nos fuerzan a reflexionar sobre los modelos de presentación de las artes vivas en el contexto museístico. Con el cambio de milenio observamos cómo entre los grandes museos se ha despertado un profundo interés por fórmulas eventuales y presenciales de arte, haciendo del performance un paradigma de las nuevas experiencias museísticas. En el ensayo What the body can do, Kirsten Maar (2015) analiza el cambio de modelo de producción cultural en el museo, sostenido por una doble y complementaria tendencia hacia la eventualización del espacio expositivo, la primera basada en la reconsideración y actualización del capital performático de los años 60, y la segunda, basada a partir de la inminente y omnipresente inclusión de coreografía contemporánea dentro de los programas expositivos. La progresiva migración de las artes performáticas al museo tiene como consecuencia lo que Claire Bishop $(2018,29)$ define como un retemporalización del performance, donde la esencia eventual del performance se transforma en un nuevo tiempo expositivo. Es decir, mientras que en la convención escénica el público se reúne a la hora designada para disfrutar colectivamente un per- 
formance de principio al final, el tiempo expositivo hace que el performance esté a disposición del espectador durante las horas de apertura del museo. En este sentido observamos cómo la imposición de una estética de la experiencia del museo está transformando la singularidad del evento performático. Si un performance se ha definido como un encuentro espacio-temporal entre performer y público, esa idiosincrasia del encuentro efímero del performance está perdiendo consistencia ontológica en favor de otros mecanismos que se adaptan más efectivamente a las necesidades del formato expositivo. En esta dirección, el performance de larga duración se viene consolidando como una modalidad paradigmática de la nueva producción performática. Tal y como nos hace reflexionar Hetzel en su propuesta, en favor de la eficiencia del performance, el encuentro deviene en un acto de co-presencia asimétrica, en la que el performance - y los performers - devienen en producto de consumo para el público, y como tal, han de estar disponibles para que el consumidor tenga una experiencia óptima del producto, amoldando su obra a los horarios de apertura y clausura de las galerías. Como acertadamente expone Sven Lüttincken $(2015,96)$, "si el capitalismo fordista bloqueaba el proceso de producción detrás de las puertas de las fábricas, el capitalismo contemporáneo organiza con frecuencia espectáculos de trabajo"t.

Desde la galería escuchamos el mismo mantra temporal que escuchábamos en la calle, sin embargo, entendemos que el sonido proviene del espacio tras la pared donde cuelga el retrato, y el pasillo lateral nos invita a continuar nuestro recorrido.

\section{Room 3. TIME-MACHINE}

Al acceder al siguiente contenedor se produce un cambio brusco en la iluminación y el diseño espacial. El contenedor está dividido en dos partes por un enorme cristal, aislando a la performer en su espacio de trabajo. En el espacio reservado para en público, un pequeño banco nos invita a contemplar el desarrollo del performance. Una chica asiática absolutamente concentrada y con la vista perdida se revela como la persona que ejecuta el tiempo:

At the next tone the time will be seventeen hours, four minutes and thirty seconds. "Ding"

At the next tone the time will be seventeen hours, four minutes and forty seconds. "Ding" 
At the next tone the time will be seventeen hours, four minutes and fifty seconds. "Ding"

At the next tone the time will be seventeen hours and fifth minutes. "Ding"

At the next tone the time will be seventeen hours, fifth minutes and ten seconds. "Ding"

At the next tone the time will be seventeen hours, fifth minutes and twenty seconds. "Ding"

Sentada frente a una mesa, un timbre de recepción y un micrófono son sus únicas herramientas de trabajo. Su voz se amplifica por todo el espacio. Tras ella, instalado en la pared desnuda del contenedor aparece un reloj digital que testifica la exactitud y precisión de su labor. Al concluir cada frase, sistemáticamente golpea el timbre. Los diez segundos que dura el intervalo de tiempo es tan breve que no le da tiempo a bajar el brazo, manteniéndolo encima del timbre siempre preparado para ejecutar el siguiente toque. Durante las ocho horas que está abierto al público el proyecto, una persona incesantemente ${ }^{5}$ recita el tiempo exacto, en una suerte de reloj humano que genera una situación entre enfermiza y fascinante. La aptitud de las performers es absolutamente mecánica, sin intuirse ningún tipo de fatiga física o psíquica. Como espectador, contemplar la cautivadora acción genera una evidente fricción entre las temporalidades objetivas y subjetivas que se nos están ofreciendo.

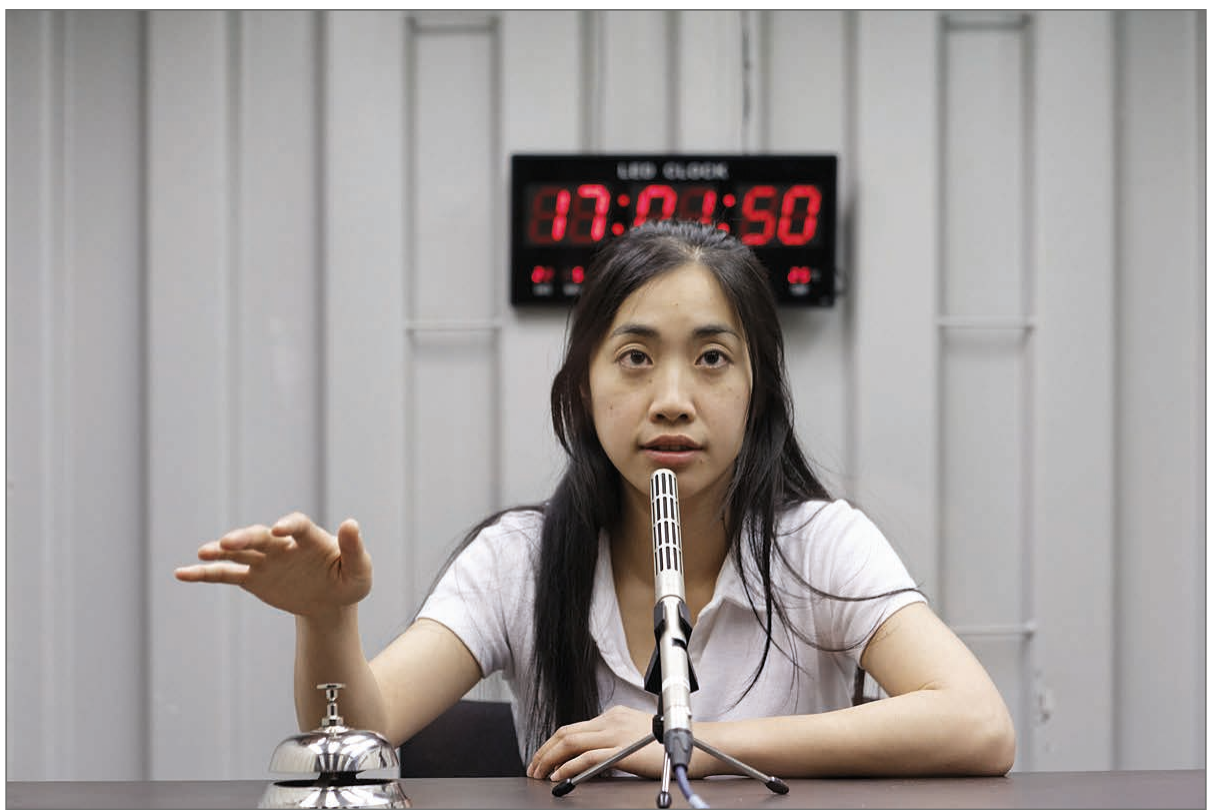

Fig. 2. Still - The Economy of Waiting, (2014). Julian Hetzel. Imagen de la instalación. (C) Thomas Leden. 
Este encuentro nos fuerza a reflexionar una vez más sobre la profesionalización, externalización y delegación de la práctica performática como un nuevo mecanismo de producción cultural. Dichos procesos implican la mercantilización del performance como producto de consumo cultural, convirtiendo al performer en la nueva fuerza obrera, ya que son sus cuerpos los que han de soportar los rigores de la adaptación al contexto performático, haciéndonos entender que la exposición de su trabajo se está convirtiendo en un nuevo capital cultural. Bishop $(2018,32)$ nos recuerda: "La forma en que las artes performáticas se acomodan al tiempo de exhibición es sobre todo mediante la repetición - de guiones, gestos o movimientos-en un bucle en vivo durante la jornada laboral"6.

\section{ROOM 4. WAITING ROOM}

Nuestro siguiente encuentro nos lleva a una improvisada sala de espera. Suelo cubierto por una moqueta azul, cinco sillas alineadas, música de ascensor, un dispensador de agua, luces fluorescentes en el techo y una persona uniformada $^{7}$ nos esperan en nuestra siguiente estación. Al entrar en este nuevo contenedor, una nueva performer nos da la bienvenida, invitándonos a tomar asiento. A diferencia del espacio anterior, donde el rol de las performers era comunicativamente distante e indiferente al público, las performers en este espacio fueron invitadas a interactuar de forma activa con los visitantes. Su misión principal era hacer esperar al público. Dicho espacio funcionaba como una situación bisagra que conectaba las dos siguientes propuestas, las cuales se planteaban como experiencias eminentemente individuales. Es decir, mientras que en los tres primeros espacios que estamos describiendo se ofrecía al público la posibilidad de parar, retroceder y contemplar, gestionando el tiempo de la visita a nuestro antojo, la performer en esta sala te invitaba a esperar, siendo en ese momento ella la persona que gestionaba el tiempo del público.

Al llegar a la sala de espera a todos los visitantes se les ofrecía un vaso de agua. Al tomar asiento podíamos observar cómo en el espacio había otras dos puertas diferentes a la que nosotros habíamos usado para entrar al contenedor. Sobre la última de las puertas había una la luz roja. Usualmente había varias personas esperando, generando una situación incómodamente absurda de estar esperando algo que no sabes muy bien qué es junto a completos extraños que tampoco saben a qué están esperando. La performer improvisaba su papel como gestora del aburrimiento: se quitaba los zapatos, bostezaba, se tumbaba en el suelo a descansar, fijaba la mirada en algunos de los visitantes, nos volvía a ofrecer agua, etc., en un comportamiento un tanto neurótico y 
divertidamente alienante. Una de las puertas se abre, y sale un miembro del público al que se le invita de nuevo a tomar asiento. La performer dirige a otra persona a pasar a la siguiente sala. La luz roja se apaga y la performer invita a un tercer visitante a pasar a la última sala. El orden por el que la performer canaliza a los visitantes no siempre sigue una lógica de llegada, sino que por el contrario juega con los tiempos de espera de los visitantes, haciendo esperar varios turnos a algunos visitantes, mientras que otros son invitados a entrar en las salas tan pronto como se quedan libres, generando una situación kafkiana. Finalmente llega nuestro momento y nos hacen pasar al quinto espacio.

\section{ROOM 5. THE POND}

Al entrar por la puerta nos encontramos de nuevo una realidad radicalmente diferente a la que acabamos de participar. En este espacio nuestra perspectiva está perfectamente delimitada a un punto de vista específico. Ligeramente elevados por una plataforma de madera, un banco está dispuesto para que disfrutemos de la experiencia contemplativa que Hetzel nos propone. En un alarde escenográfico, el contenedor —-mucho más largo que el resto- ha sido completamente transformado en un paisaje onírico. El suelo está completamente cubierto por una lámina de agua desde la que crecen esporádicamente islas vegetales que nos hace pensar en un humedal. Tanto las esquinas superiores como el fondo del espacio han sido diseñados como una estructura cóncava, generando la sensación de estar dentro de un espacio tubular infinito. Un cuidado diseño de luces va transformando delicadamente los tonos y temperatura de la luz, efecto que se refuerza por el uso de humo en el espacio, generando brumas y difuminando un paisaje que permanece en constante transformación. Al fondo del espacio, aparece una persona estática con los pies dentro del agua. Es la figura de un hombre vistiendo un traje de chaqueta, dándonos la espalda y mirando al infinito. El contraste que genera esta figura contemporánea en este paisaje onírico y atemporal, puede llegar a recordarnos a los paisajes alegóricos realizados por Caspar David Friedrich. El paisaje visual se ve implementado con la construcción de un paisaje sonoro que nos transporta a una naturaleza acuosa y crepuscular poblada de insectos. Como propuesta contemplativa, el público es libre de permanecer en este ecosistema el tiempo que estime oportuno, ofreciendo el espacio para emplear nuestro tiempo de una manera radicalmente ociosa y ralentizada. 


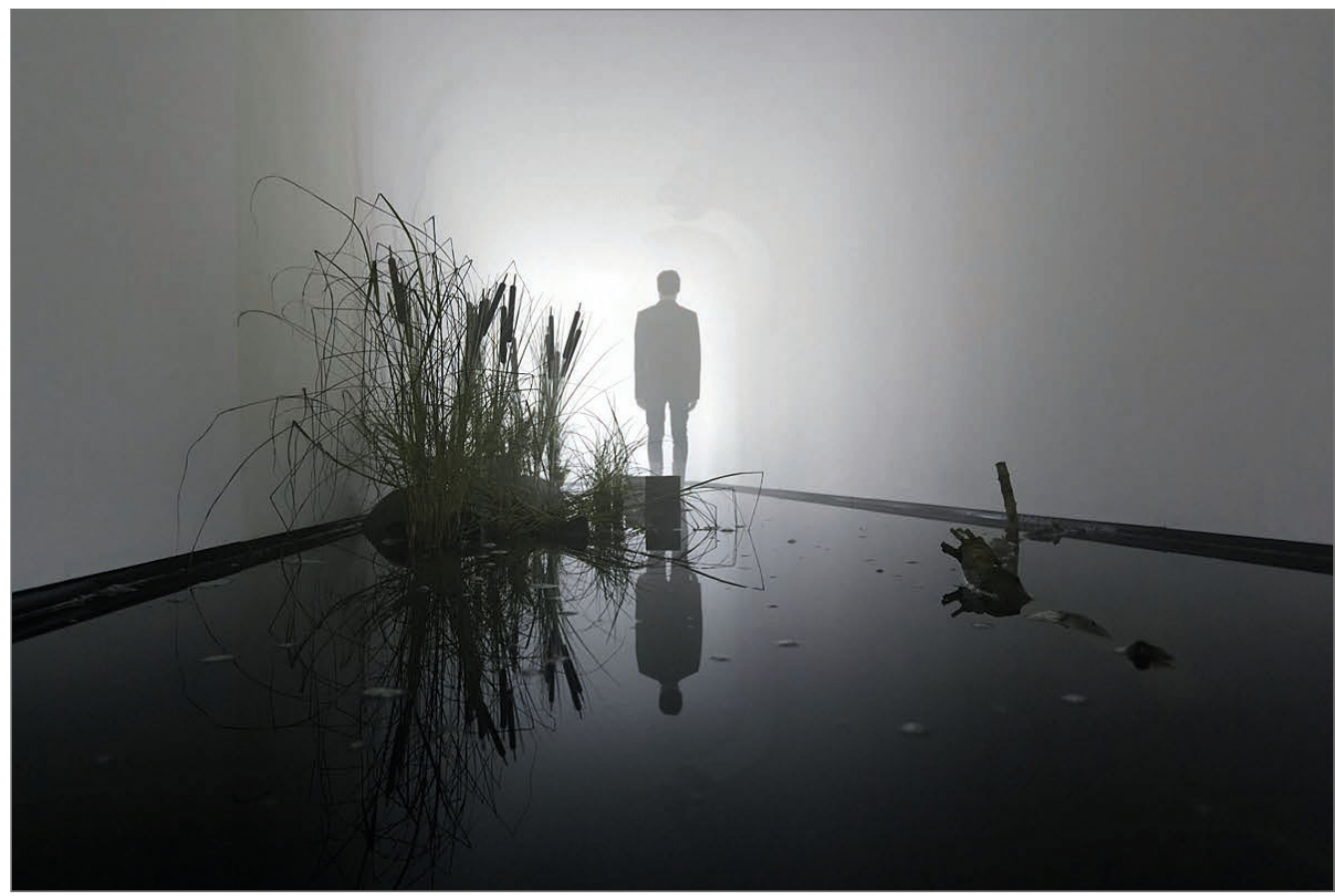

Fig. 3. Still — The Economy of Waiting, (2014). Julian Hetzel. Imagen de la instalación. @ Thomas Leden.

\section{Room 6. Nothing For Something}

Una vez que hemos regresado a la sala de espera, y finalmente somos invitados a pasar a la última sala, Hetzel vuelve a sorprendernos. El último espacio vuelve a transformarse en una galería de arte contemporáneo, con suelo, techo y paredes blancas y brillantes. Un banco organiza y divide el espacio en dos. Al fondo, una persona nos está esperando sentada en el suelo. Junto al banco, en la pared una cartela expositiva nos da indicaciones de la propuesta:

\section{Nothing for Something}

Wood, Paint, TL-Lights, Hygrothermometer, Bench, Homeless person.

For the execution of his performance, the homeless person receives 8,50 Euro per hour as a donation, an amount of money which is equivalently valued to the current minimum wage in the Netherlands. 
This is a meeting with the Self through an encounter with the Other.

El encuentro con una persona sin hogar es el elemento central de esta instalación. Tal y como indica la cartela expositiva, a la persona que cada día es invitada a participar en la instalación - a lo largo de las dos semanas fueron tres los colaboradores - se le ofrece una compensación económica de 8,50 euros por hora en concepto de donación. Dicha compensación equivale al salario mínimo de 2014 en los Países Bajos. Su función consistía en pasar tiempo dentro del espacio. No estaban obligados a establecer contacto con los visitantes, simplemente permanecer, esperar, pasar tiempo. Es decir, se les invitaba a capitalizar su función rutinaria, pero ofreciéndoles un nuevo contexto compuesto por un marco espacial, cultural e social que el proyecto propone. En este sentido, podríamos entender dicha propuesta como un readymade performático, donde la función de Hetzel pasa por delimitar dicho marco espacio-temporal en un encuentro que difícilmente podría suceder fuera del contenedor cultural del proyecto. En una entrevista recientemente publicada, Hetzel $(2019,84)$ reflexiona sobre las implicaciones de esta propuesta: "Me gusta desafiar al público, me gusta que se planteen cuestiones en torno a la responsabilidad y a la ética, como en Still: ¿cómo tratamos a las personas que están viviendo en las calles? Dirigiendo esta cuestión al público, este se ve forzado a negociar con dicha pregunta o situación. Creo que esto es lo que intento hacer una y otra vez"s.

La situación de este encuentro entre dos personas es enmarcada por Hetzel, pero negociado por los visitantes. La propuesta se transforma en un encuentro con uno mismo a través del encuentro con el otro. Generalmente hablando, se establecen conversaciones íntimas donde la persona sin techo reflexiona sobre cómo ha llegado a esa situación, cómo es su día a día, su situación política, cómo ve a la sociedad, y cómo piensa que la sociedad le ve. La simplicidad, sobriedad e inmaterialidad de la propuesta nos fuerza a mirar hacia su esencia: la relatividad temporal como una construcción eminentemente humana y social. Cuando decidimos que nuestra conversación ha terminado, la persona con la que acabamos de establecer una conversación sobre qué significa vivir sin techo nos invita amablemente a salir a la calle por una última puerta para finalizar nuestra visita por la instalación.

Que la mayoría de las instituciones culturales trabajen hacia modelos expositivos donde se incrementa el peso del arte como experiencia, y donde los objetos artísticos están siendo reemplazados por cuerpos vivos, solo puede 
remitirnos a un cambio en los modelos de producción de subjetividad. El objetivo no es otro que generar propuestas estéticas que alienten e impliquen a un público activo, creativo y eminentemente participativo. En este contexto nos parece lógico que empiecen a alzarse voces críticas sobre estas estrategias artísticas, ya que estos mecanismos de producción cultural no solo reemplazan el objeto artístico por una subjetividad interpersonal, sino que como nos evidencia la propuesta de Hetzel, el objeto artístico está siendo reemplazado por cuerpos humanos. Dicho de otro modo, la plasticidad intersubjetiva de la propuesta de Hetzel se construye a partir de una relación laboral y profesional, mano de obra que circula en el mercado del arte, con lo que nos acercamos peligrosamente a los modelos productivos propios del neoliberalismo. Como acertadamente plantea Diego Albarrán (2017, 32): "algunos de los elementos emancipadores que el artista parece potenciar, tales como la creatividad, la capacidad de negociar situaciones imprevisibles, la aparente precariedad material y el carácter casual y desenfadado de sus propuestas, son elementos claves en los modos de subjetivación del capitalismo postindustrial, empeñado en convertir cada momento de nuestras vidas en tiempo productivo".

\section{CONCLUSIONES}

El viaje temporal, emocional y político que experimentamos en el recorrido por las experiencias (Alston) que propone de Still - The Economy of Waiting, trata de hacer visible lo aparente. La multiplicidad de ángulos y perspectivas que se establecen en las diferentes instalaciones y encuentros que Hetzel nos propone, posibilita el espacio para hacernos reflexionar sobre el uso del tiempo en nuestra acelerada realidad postindustrial y globalizada, proponiendo modelos de desaceleración y alternativas temporales que nos hagan reflexionar sobre nuestra relación con un capitalismo neoliberal en constante movimiento.

Como observamos en nuestro caso de estudio Still - The Economy of Waiting, Hetzel investiga una delicada relación entre los modelos productivos y el uso del tiempo en nuestra sociedad contemporánea. La flexibilidad temporal y las estructuras duracionales son utilizadas como estrategias performáticas con la capacidad de generar un impacto directo en el público, ya que como alternativas temporales constituyen un acto de denuncia en si mismo, forzándonos a adoptar una postura crítica. En esta línea argumental, y a modo de conclusión, podríamos defender que bien sea en la presencia o ausencia de 
performers, en sus acciones o pasividad, desde las estrategias temporales colectivas o individuales, observamos cómo la flexibilidad y vacío temporal que nos propone este trabajo solo puede ser ocupado por el posicionamiento ético del público. En el análisis de lo temporal que hemos desarrollado en este ejemplo, podemos apreciar cómo Hetzel se aleja decididamente de preocupaciones formales o disciplinares en torno a lo temporal, para por el contrario centrarse en proponer alternativas performáticas que nos hagan reflexionar sobre nuestra posición personal y social en un contexto histórico y político que nos ha tocado vivir.

\section{Referencias bibliográficas}

Albarrán Diego, Juan. 2017. "Sehgal no invita a la lógica: Performance, experiencia y economía inmaterial en Tino Sehgal". Sin Objeto 00: 24-39

Alston, Adam. 2013. "Audience participation and neoliberal value: Risk, agency and responsibility in immersive theatre". Performance Research 18(2): 128-38

Bergson, Henri. (1907) 1998. Creative revolution. Nueva York: Dover

Bishop, Claire. 2018. "Black Box, White Cube, Gray Zone: Dance exhibitions and audience attention". TDR: The Drama Review 62(2): 22-42

Hernández-Navarro, Miguel Ángel. 2008. "Presentación: Antagonismos temporalis". En Heterocronías: Tiempo, arte y arqueología del presente, Miguel Ángel Hernández-Navarro, ed. Murcia: CENDEAC

Hetzel, Julian. 2019. "Keep it real: A conversation between performance artist Julian Hetzel and Theresa Schütz". En Staging spectators in immersive performances: Commit Yourself!, Doris Kolesch, Theresa Schütz \& Sophie Nikoleit, eds. London: Routledge

Lehmann, Hans-Thies. (1999) 2017. Teatro posdramático. Traducción, Diana González y otros. Murcia: CENDEAC

Lütticken, Sven. 2015. "Dance factory”. Mousse 50: 90-101. https: //svenlutticken.files.word. com/2014/05/mm_50_lutticken_doppie.pdf

Maar, Kirsten. 2015. "What the body can do: Reconsidering the role of the moving body in exhibition contexts". Stedelijk Studies \#3. https: //stedelijkstudies.com/journal/what-a-bodycan-do/

Steyerl, Hito. 2016. "Art as occupation: Claims for an autonomy of life". E-flux Journal \#30. www.e-flux.com/journal/30/68140/art-as-occupation-claims-for-an-autonomy-of-life/

Weeks, Kathi. 2013. "The problem with work". En Living labor, Milena Hoegsberg \& Cora Fisher, eds. Berlin: Sternberg

\section{Notas}

\footnotetext{
${ }^{1}$ Traducción del autor, texto original: "The shift from work to occupation applies in the most different areas of contemporary daily activity. It marks a transition far greater than the
} 
often-described shift from a Fordist to post-Fordist economy. Instead of being seen as a means of earning, it is seen as a way of spending time and resources. It clearly accents the passage from an economy based on production to an economy fuelled by waste, from time progressing to time spent or even idled away, from a space defined by clear divisions to an entangled and complex territory. Perhaps most importantly: occupation is not a means to an end, as traditional labor is. Occupation is in many cases an end in itself".

${ }^{2}$ Su traducción al español vendría a ser: Inmóvil. La economía de la espera.

${ }^{3}$ Dado lo universal de este clásico del arte conceptual, no entendemos necesario entrar a describirlo en este apartado. De cualquier modo, para contrastar la información que aquí exponemos hemos recurrido a la entrada de la obra en la website del Museo Nacional Centro de Arte Reina Sofia de Madrid. Recurso digital disponible en: https://www.museoreinasofia.es/coleccion/obra/one-and-three-chairs-tres-sillas [visitado el 31-05-2019].

${ }^{4}$ Traducción del autor, texto original: "If Fordist capitalism locked away the production process behind factory gates, contemporary capitalism frequently stages spectacles of labor".

${ }^{5}$ En el desarrollo del performance se contó con la participación de dos performers, Miri Lee y Wen Chin Fu, que ejecutaban la acción en intervalos de veinte minutos.

${ }^{6}$ Traducción del autor, texto original: "The way in which the performing arts accommodate themselves to exhibition time is above all by repetition - of scripts, gestures, or movements on a live loop for the duration of the working day".

${ }^{7}$ En la presentación en Utrecht se contó con la participación de Nadia Amin, Mariangela Tinelli, Matra Zisolek, Agustina Muñoz y Esther Mungambi.

${ }^{8}$ Traducción del autor, texto original: "I like to challenge the audience, I like to open up questions about responsibility, about ethics, like in Still: how do we treat people who're living on the streets? By redirecting these questions to the audience everybody has to negotiate these questions or situations. I think this is what I'm trying to do again and again". 